\title{
Commentary: Bettering BCG: a tough task for a TB vaccine?
}

\author{
Brahm S. Srivastava ${ }^{1}$, Vipul K. Singh ${ }^{2 *}$, Vivek K. Kashyap ${ }^{3}$, Ranjana Srivastava ${ }^{1}$, \\ Arshad Khan ${ }^{2}$ and Chinnaswamy Jagannath ${ }^{2}$ \\ ${ }^{1}$ Nextec Lifesciences, Lucknow, India, ${ }^{2}$ Department of Pathology and Genomic Medicine, Houston Methodist Research \\ Institute, Houston, TX, United States, ${ }^{3}$ Department of Immunology and Microbiology, University of Texas Rio Grande Valley, \\ McAllen, TX, United States
}

Keywords: LipY lipase from Mycobacterium tuberculosis, BCG-bacille calmette-guérin vaccine, M. tuberculosis, vaccine, booster antigen

\section{OPEN ACCESS}

Edited by:

Denise Doolan,

James Cook University, Australia

Reviewed by:

Arun Kumar,

Coalition for Epidemic Preparedness

Innovations (CEPI), Norway

Camille Locht,

Institut National de la Santé et de la

Recherche Médicale

(INSERM), France

${ }^{*}$ Correspondence:

Vipul K. Singh

vsingh@houstonmethodist.org

Specialty section:

This article was submitted to

Vaccines and Molecular Therapeutics,

a section of the journal

Frontiers in Immunology

Received: 22 May 2019

Accepted: 30 August 2019

Published: 12 September 2019

Citation:

Srivastava BS, Singh VK, Kashyap VK,

Srivastava $R$, Khan A and

Jagannath $C$ (2019) Commentary:

Bettering BCG: a tough task for a TB

vaccine? Front. Immunol. 10:2195.

doi: 10.3389/fimmu.2019.02195

\section{A Commentary on}

Bettering BCG: a tough task for a TB vaccine?

by Bishai, W., Sullivan, Z., Bloom, B. R., and Andersen, P. (2013). Nat. Med. 19, 410-411. doi: $10.1038 / \mathrm{nm} .3153$

The BCG (Bacille Calmette-Guerin) vaccine is the only licensed vaccine for tuberculosis (TB), with several million children vaccinated to date. BCG protects children against disseminated TB, but shows variable efficacy in adults against pulmonary TB. Multiple efforts have been undertaken to improve BCG but have had unsatisfactory results, although these variations have largely been tested only in animal models of TB. These efforts have included: (i) recombinant BCG (rBCG) expressing cytokines, (ii) putative protective antigens derived from Mycobacterium tuberculosis, and (iii) subunit antigens purported to add immunogenic "value" to BCG (1).

In an attempt to improve BCG, a modified vaccinia virus Ankara expressing antigen 85A (MVA85A) was administered to previously BCG-vaccinated infants and young children in a clinical trial conducted in South Africa. Investigators were hopeful based on promising results observed in animal models of TB. However, the booster vaccine failed to protect vaccine recipients from infection with $M$. tuberculosis (2). Bishai et al. expressed disappointment with the outcome of the trial (3). Nevertheless, they expressed hope and proposed some issues for future consideration. The results of this trial emphasize the need for identification of biomarkers that correlate with protection and active TB in young and adult human subjects, which should be the gold standard by which the efficacy of new vaccines are evaluated. Selection of young BCG-vaccinated children to test the efficacy of MVA85A has also been questioned. Since, TB is a lung disease that occurs in the adult population, conducting a trial for booster vaccines may be more relevant in BCG-vaccinated adolescents (15+ years old). Bishai et al. propose to conduct any future trials of booster vaccines in adolescent and/or adult population, infected with active or latent M. tuberculosis (3), especially in Indian and African continents where TB is endemic. The logic of selection of modified vaccinia virus as a carrier of antigen $85 \mathrm{~A}$ is also intriguing, since the world's population has received smallpox vaccinations. Whether the engineered vaccinia vaccine MVA85A will be able to survive and replicate in a vaccinated population long enough to induce sufficient immunity is questionable. 


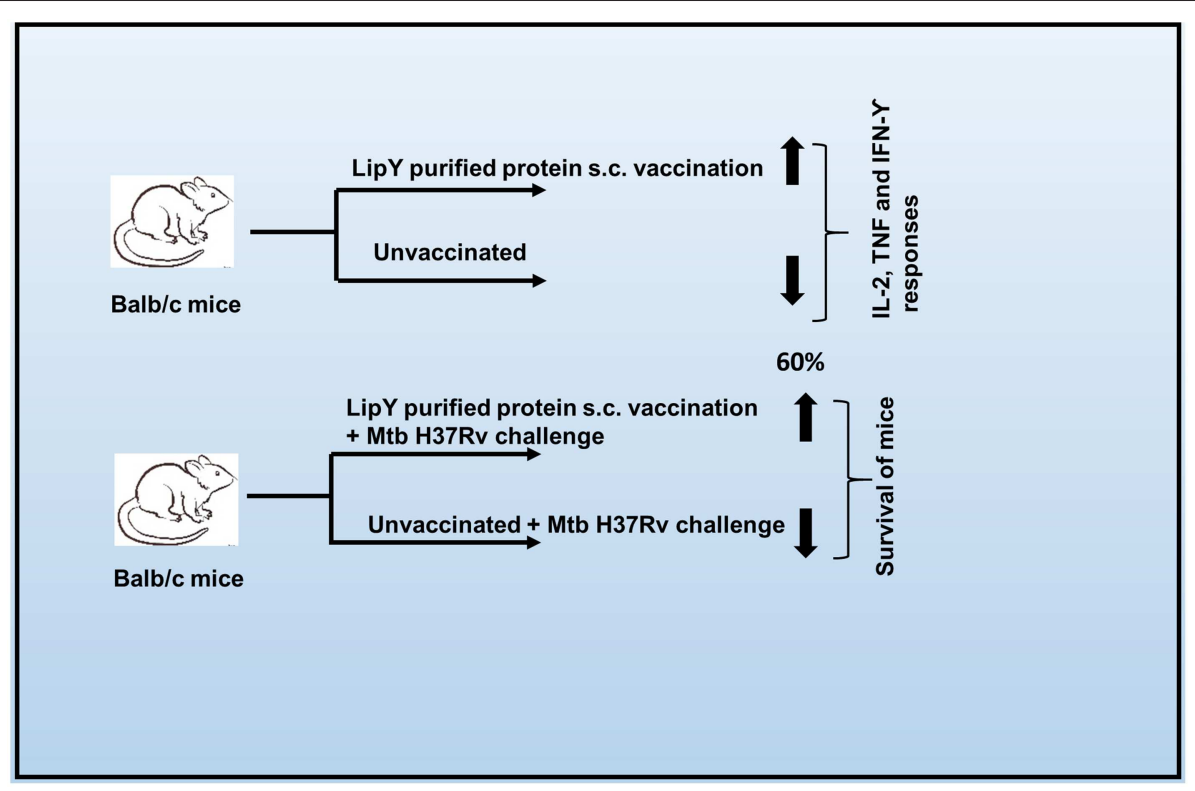

FIGURE 1 | Potential role of LipY as a vaccine candidate for protection against tuberculosis.

Despite these setbacks, there are new candidates in the pipeline currently being evaluated. In our quest for a booster antigen, we have studied Rv3097c of M. tuberculosis encoding a lipase (LipY) in a mouse model as a protective antigen to counter infection of M. tuberculosis $(4,5)$.

When the Rv3097c gene was overexpressed from a plasmid in BCG, the recombinant BCG lost immunogenicity. That is, mice immunized with recombinant BCG and challenged with $M$. tuberculosis were sensitive to killing just like naïve, unimmunized mice. We found over-expression of LipY caused suppression of the protective host immune response (Th1) and the rise of the immunosuppressive Th2 response (4). Mice died rapidly with reduced expression of cytokines and interleukins (4). Similarly, when Rv3097c gene was overexpressed in M. tuberculosis, recombinant $M$. tuberculosis was more virulent than wild-type M. tuberculosis. The mean survival time of infected mice was reduced, the bacillary load was higher, and lung pathology was severe. However, mice immunized with recombinant, purified LipY were protected against challenge with $M$. tuberculosis, and this correlated with an effective immune response (5). As the LipY lipase is a cell wall-associated protein that interacts with effector molecules of the immune system (6), immunization with subunit antigen LipY could generate an immune response against mycobacterial lipase. LipY and other similar lipolytic enzymes could therefore be explored as potential adjuncts to BCG or new therapeutic vaccine candidates against mycobacterial infections (Figure 1) (5).

Non-tubercular mycobacteria (NTM) have also been known to cause a spectrum of diseases in humans, although with less severe pathogenesis as compared to M. tuberculosis. M. fortuitum, which is an NTM, has been historically shown to cause opportunistic secondary infections in humans. Mice are susceptible to infection of $M$. fortuitum, and display disease symptoms, but infection only causes mortality in $25-30 \%$ of cases. Bacilli lodge in the kidney, where they multiply and cause symptoms (7). Bioinformatic analysis indicates that the M. fortuitum genome has no Rv3097c gene or its homolog as confirmed by sequence analysis and western blot (unpublished data). When Rv3097c gene was expressed in M. fortuitum, mortality of mice infected with recombinant $M$. fortuitum increased to $100 \%$ compared to $25-30 \%$ with wild-type $M$. fortuitum infected mice. Thus, LipY lipase can modulate the virulence of $M$. tuberculosis and NTM M. fortuitum by downregulating the host immune response.

The published literature and our analyses suggest that mycobacterial LipY lipase is a cell wall-associated enzyme, which also acts as a virulent factor in M. tuberculosis. LipY lipase has an important function in the biology of mycobacteria. It causes catabolism of stored triacylglycerol, thus releasing free fatty acids as a "lipid diet" for starving mycobacteria during latency $(8,9)$. LipY is up-regulated in various conditions and environments. We measured gene expression of LipY using real-time PCR in various in vitro, ex vivo, and in vivo conditions. LipY expression was enhanced in infected mouse macrophages (10) and in the lungs of infected mice (11). An increase in expression of LipY was observed in cells grown in vitro in oxygen and nutrient-depleted conditions that mimic dormancy (12). Therefore, environmental stress induces an increase in transcription of the LipY gene (Rv3097c). Interestingly, inhibitors of LipY were identified in our lab that inhibited the growth of the bacilli under hypoxic conditions, but not that of aerobically-grown cultures (13).

In conclusion, there has been much effort invested in augmenting the BCG vaccine, with various and often disappointing results. The LipY lipase is an important antigen of M. tuberculosis that could be explored to boost the BCG vaccine and improve protection against TB. 


\section{AUTHOR CONTRIBUTIONS}

BS wrote the manuscript and VS edited the manuscript. VK, RS, $\mathrm{AK}$, and $\mathrm{CJ}$ provided the suggestions and comments to improve the manuscript.

\section{REFERENCES}

1. Singh VK, Srivastava R, Srivastava BS. Manipulation of BCG vaccine: a double-edged sword. Eur J Clin Microbiol Infect Dis. (2016) 35:535-43. doi: 10.1007/s10096-016-2579-y

2. Tameris MD, Hatherill M, Landry BS, Scriba TJ, Snowden MA, Lockhart S, et al. Safety and efficacy of MVA85A, a new tuberculosis vaccine, in infants previously vaccinated with BCG: a randomised, placebo-controlled phase $2 \mathrm{~b}$ trial. Lancet. (2013) 381:1021-8. doi: 10.1016/S0140-6736(13)60177-4

3. Bishai W, Sullivan Z, Bloom BR, Andersen P. Bettering BCG: a tough task for a TB vaccine? Nat Med. (2013) 19:410-1. doi: 10.1038/nm.3153

4. Singh VK, Srivastava V, Singh V, Rastogi N, Roy R, Shaw $\mathrm{AK}$, et al. Overexpression of Rv3097c in Mycobacterium bovis BCG abolished the efficacy of BCG vaccine to protect against Mycobacterium tuberculosis infection in mice. Vaccine. (2011) 29:4754-60. doi: 10.1016/j.vaccine.2011.04.086

5. Singh VK, Srivastava M, Dasgupta A, Singh MP, Srivastava R, Srivastava BS. Increased virulence of Mycobacterium tuberculosis H37Rv overexpressing LipY in a murine model. Tuberculosis. (2014) 94:252-61. doi: 10.1016/j.tube.2014.02.001

6. Mishra KC, de Chastellier C, Narayana Y, Bifani P, Brown AK, Besra GS, et al. Functional role of the PE domain and immunogenicity of the Mycobacterium tuberculosis triacylglycerol hydrolase LipY. Infect Immun. (2008) 76:127-40. doi: 10.1128/IAI.00410-07

7. Parti RPS, Srivastava S, Gachhui R, Srivastava KK, Srivastava R. Murine infection model for Mycobacterium fortuitum. Microbes Infect. (2005) 7:34955. doi: 10.1016/j.micinf.2004.11.006

8. Deb C, Daniel J, Sirakova TD, Abomoelak B, Dubey VS, Kolattukudy PE. A novel lipase belonging to the hormone-sensitive lipase family induced under starvation to utilize stored triacylglycerol in Mycobacterium tuberculosis. J Biol Chem. (2006) 281:3866-75. doi: 10.1074/jbc.M5055 56200

\section{ACKNOWLEDGMENTS}

We thank Dr. Kathryn Stockbauer for assisting in editing the English and improving the language of the manuscript.

9. Low KL, Rao PSS, Shui G, Bendt AK, Pethe K, Dick T, et al. Triacylglycerol utilization is required for regrowth of in vitro hypoxic nonreplicating Mycobacterium bovis bacillus Calmette-Guerin. J Bacteriol. (2009) 191:503743. doi: 10.1128/JB.00530-09

10. Srivastava V, Rouanet C, Srivastava R, Ramalingam B, Locht C, Srivastava BS. Macrophage-specific Mycobacterium tuberculosis genes: identification by green fluorescent protein and kanamycin resistance selection. Microbiology. (2007) 153:659-66. doi: 10.1099/mic.0.2006/000547-0

11. Srivastava V, Jain A, Srivastava BS, Srivastava R. Selection of genes of Mycobacterium tuberculosis upregulated during residence in lungs of infected mice. Tuberculosis. (2008) 88:171-7. doi: 10.1016/j.tube.2007.10.002

12. Saxena A, Srivastava V, Srivastava R, Srivastava BS. Identification of genes of Mycobacterium tuberculosis upregulated during anaerobic persistence by fluorescence and kanamycin resistance selection. Tuberculosis. (2008) 88:51825. doi: 10.1016/j.tube.2008.01.003

13. Saxena AK, Roy KK, Singh S, Vishnoi SP, Kumar A, Kashyap VK, et al. Identification and characterisation of small-molecule inhibitors of Rv3097cencoded lipase (LipY) of Mycobacterium tuberculosis that selectively inhibit growth of bacilli in hypoxia. Int J Antimicrob Agents. (2013) 42:27-35. doi: 10.1016/j.ijantimicag.2013.03.007

Conflict of Interest Statement: The authors declare that the research was conducted in the absence of any commercial or financial relationships that could be construed as a potential conflict of interest.

Copyright (c) 2019 Srivastava, Singh, Kashyap, Srivastava, Khan and Jagannath. This is an open-access article distributed under the terms of the Creative Commons Attribution License (CC BY). The use, distribution or reproduction in other forums is permitted, provided the original author(s) and the copyright owner(s) are credited and that the original publication in this journal is cited, in accordance with accepted academic practice. No use, distribution or reproduction is permitted which does not comply with these terms. 